\title{
Realization of the Regional Advantageous Agricultural Industries Analysis System
}

\author{
Kaimeng Sun \\ Institute of Agricultural Information, Chinese Academy of Agricultural Sciences, \\ Beijing, P.R. China 100081
}

\begin{abstract}
In this paper, a system for analyzing the strategic adjustment of regional agricultural industries in China is briefly introduced, particularly on several system functions of database, region selection, statistics, comparative analysis and result presentation in terms of their technical realizations. The introduction is focused on the realized comparative advantageous agricultural analysis computation methods and assessment recommendations. From this paper, we can see an application of a knowledge based system for regional advantageous agriculture analysis. Technical process of system analysis and system design for developing such a knowledge-based system is presented.
\end{abstract}

Keywords: GIS, Agricultural regions, Computer, System.

\section{Introduction of the System}

\subsection{Objectives}

The Regional Advantageous Agricultural Industries Analysis System (or the System hereafter) is designed to analyze country-based advantageous agro industries and their indicators based on a region's natural resources, current agricultural production, and agro-economic performances in China. Through analyzing cross-regional (neighboring regions and within the province) comparative advantages, current regional development will be assessed. Meanwhile, by using temporal data analysis, development of the regional advantages can be obtained so as to identify the long-term trends of the regional economy. Therefore, the System is designed by focusing on regional advantages to be taken. However, due to unavailable agricultural economic data sets, some regional industrial advantages may not be fully analyzed. Data in this study have included that of regional natural resources, labor force, major crop production, and local enterprises. By analyzing these datasets, the overall development of regional advantageous agricultural industries can be understood.

\subsection{Major System Modules}

By considering data flow, functional requirements and system characteristics, the System is designed with modules of data management, data processing, knowledge reasoning, result output, and man-machine interface. Working together, a complete system flow from data selection, model computation, inference, and analysis result 
output is realized to obtain the overall analysis and assessment of regional agriculture economy.

Therefore, the System is composed of region selection, statistics analysis, advantage comparative analysis, assessment, and data management functional modules.

\section{Realization of Database System}

In order to precisely define the regional industries, large amount of basic data are necessary. The analysis results rely heavily on availability of accuracy of detailed information on resources, agricultural production, and local economic activities. For this purpose, relevant databases are developed containing spatial, temporal, economic, and knowledge. Databases in the System are classified into five areas:

\subsection{Spatial Database}

In the regional advantageous agricultural industrial analysis system, a 1:4,000,000 national country spatial database developed by Super Map is used; including the map referenced spatial attributes and county names and codes. Using this spatially relational database, county level spatial locations and related agricultural basic agroeconomy data are utilized to extract datasets for functional modules for statistics, computation, analysis, and assessment.

\subsection{Agricultural Economy Basic Database}

According to the System design, a total of 64 datasets are selected on agricultural resources, crop plantation of both grain crops and cash crops, livestock farming, local enterprises, and economic indicators, and as well as planting areas, population, stable harvesting area, summer crop plantation area and summer crop yield. All the data are original and maintained in ACCESS data tables.

\subsection{Interim and Final Computation Result Database}

The database is developed by ACCESS, including:

Interim computation results: Storage of agricultural economic original data, such as wheat planting area, total yield, for ranking and comparative advantages computation.

Computation results: Storage of computation results such as statistics and comparative advantages computation results.

\subsection{Knowledge Based Database}

It is used to store knowledge for reasoning computation. This information set is basis for inference and rules for analysis and judgment. So it is like a combination of knowledge base and rule base.

\subsection{Code Reference Database (Data Dictionary)}

It contains data dictionary to be used in the system, including names of provinces, counties, project title, menu, and database field references. 


\section{Realization of Data (Region) Selection Module}

\subsection{Region Selection on Map}

The GIS Supermap platform is used to select a region. After a region is selected on map, the program will create a dataset for the selected region including region name and code. The two data attributes are accessed to query matched agricultural data. According to the design, simple statistics will be computed such as unit crop yield and computation results will be exported into the result database for future use. Once a region is selected by user, the region will be marked red. Relevant adjacent regions will be selected by user.

\subsection{Region Selection by Name}

User can choose a province name at first and the system will automatically display related counties in a popup. According to user selection in the popup, system will extract region code from county database and then access corresponding spatial database to the county and finally mark red of the selected region on the map. Meanwhile, the system will provide simple statistics such as crop yields and then store the computation result in the database for future function use.

\subsection{Adjecient Region Selection}

To select an adjacent region is to select border regions with the selection region, with common border on the map. The selection of adjacent region is based on user selection and extracted from spatial analysis. The selected province is based on the first two digits of region code (provincial code). Agricultural data are extracted from agro-economy database according to the first two code digits automatically by the system.

\section{Statistics Module}

Statistics computation includes ranking of the indices of the region by comparing with adjacent regions. The ranking and computation method is not presented in detail here.

For overall status assessment, method can be different for selected and adjacent regions. For six classes of data such as agricultural resources, grain crop plantation, cash crop plantation, animal farming, rural enterprises, and economic indices, items can be different for different regions. The overall assessment in terms of excellent, good, moderate, poor, or extremely poor will be resulted.

To achieve the overall assessment, according to selected and adjacent regions and their indices, rankings for the indices will be computed, including 6 items of agricultural resources, 8 items of grain crop indices, 7 items of cash crop yields, 10 items of animal farming indices, 5 items of rural enterprises, and 9 items of major economy indices. 


\section{Omparative Advantages Computation}

Computation of comparative advantages of a region is firstly focused on agricultural crop indices such as for summer crops, wheat, corn, beans, oil crops, and sweet beet. Scale production advantages, productivity advantages, and efficiency advantages will be firstly computed and then the overall advantages based on their squares and square roots. For other non-crop indices, only scale advantages will be computed such as arable land area, rural population, paddy land area, rural enterprises, rural enterprise employment, total income, etc. Therefore different processing methods are used for the two different types of data.

The computation method for comparative advantages is detailed as follows:

\subsection{Scale Advantages}

An index of a selected region (county), such as crop plantation area, fresh water fishing, or enterprises, is compared with mean of adjacent regions to represent its scale advantage. The formula will be:

$$
S_{i j}=\frac{A_{i j}}{\frac{1}{n} \sum_{j=1}^{n} A_{i j}}
$$

Where $S_{i j}$ is the scale advantage index for the $j$ th region and the $i$ th index; $A_{i j}$ is the value of the $j$ th region and the $i$ th index; $j$ is total number of regions (to compare with adjacent regions, it include the selected region and adjacent regions. If to compare with its province data, it include all regions in the province totaled $n$ ), $j=1,2,3, \ldots, n ; i$ is indices to be compared such as rice, wheat, corn, and other grain crops.

\subsection{Productivity Advantages}

The productivity advantages will be presented based on natural conditions of major agricultural crops and unit yield indices, i.e.:

$$
P_{i j}=\frac{Q_{i j} / A_{i j}}{\sum_{j=1}^{n} Q_{i j} / \sum_{j=1}^{n} A_{i j}}
$$

where $P_{i j}$ is productivity advantage index at $j$ th region and for $i$ th crop; $Q_{i j}$ is total production of the $i$ th crop at the $j$ th region; $Q_{i j} / A_{i j}$ represents the land productivity level at the $j$ th region for the $i$ th crop; the denominator is total number of regions (to compare with adjacent regions, it include the selected region and adjacent regions. If to compare with its province data, it includes all regions in the 
province totaled $n$ ), or the average land productivity for the $i$ th crop; $j=1,2,3, \ldots$, $n ; i$ is the comparative index to participate in the assessment.

\subsection{Efficiency Advantages}

Per capita agricultural product production at selected region is compared with the average to present production efficiency (to compare with adjacent regions, it include the selected region and adjacent regions. If to compare with its province data, it includes all regions in the province), i.e.:

$$
E_{i j}=\frac{Q_{i j} / L_{i j}}{\sum_{j=1}^{n} Q_{i j} / \sum_{j=1}^{n} L_{i j}}
$$

where $E_{i j}$ is efficiency advantage index at the $j$ th region for the $i$ th crop; $L_{i j}$ is number of labors at the $j$ th region for crop $i$; therefore $Q_{i j} / L_{i j}$ reflects the labor efficiency level at the $j$ th region for crop $i$; the denominator is the average production efficiency over $i$ crops such as rice, wheat, corn, and other gain crops (to compare with adjacent regions, it include the selected region and adjacent regions. If to compare with its province data, it include all regions in the province totaled $n$ ).

\subsection{Comparative Advantage Index}

The comparative advantage is assessed by an integrated consideration of scale advantages, productivity advantages, and efficiency advantages in a region. Two methods can be considered. If to compare with adjacent regions, it includes the selected region and adjacent regions. This is called comparative advantage with adjacent regions. If to compare with its province data, it include all regions in the province. This is called comparative advantage in the province.

$$
C_{i j}=\sqrt[3]{S_{i j} \bullet P_{i j} \bullet E_{i j}}
$$

where $C_{i j}$ is the comparative advantage index at the $j$ th region for crop $i$, representing the overall advantage for the region $j$ and crop $i$.

When the value of $S_{i j}, P_{i j}, E_{i j}$, or $C_{i j}$ is greater than, it represents the region is advantageous, otherwise no advantage. If the value $S_{i j}$ for the region is greater than 1 , it demonstrates a scale advantage in the region compared over other regions. If the value $C_{i j}$ for the region is greater than 1 , it demonstrates an overall advantage in the region compared over other regions. The greater the value is, the bigger the overall advantage, vice versa (see Nie Fengying, 2006). 


\subsection{Different Comparative Indices for Different Crops and Economic Indices}

In the agricultural basic database used in the study, some data items directly related to crop production such as yield of summer crops, rice, wheat, corn, beans, oil crops are used to compute $S_{i j}, P_{i j}, E_{i j}$, and then $C_{i j}$ is computed to present the overall comparative advantage. If the value of $C_{i j}>1$, the index in the region is called comparative advantageous over adjacent or province.

For some indices not directly related crop production, such as arable land area, rural population, rural enterprises, and agricultural income, the system will only compute their scale advantage $S_{i j}$ by comparing mean value over the adjacent regions. If the value is greater than 1 , it is scale advantageous, vice versa.

The comparative advantage computation can be for adjacent or for province. Purpose of the two methods is to represent the region's advantageous agricultural industries in the overall region. In fact, an advantage over adjacent regions may not be so over the overall province, vice versa.

\section{Output Display}

\subsection{Graphical Output Display}

Two kinds of output graphical displays in the regional advantageous industry analysis system will be provided: comparing a single index with all regions and ranking comparison in terms of each of the six index types including agricultural resources, grain crop plantation, cash crop yields, animal farming, rural enterprises, and economic performance. The comparison result can be displayed by bar chart as the most illustrative way.

\subsection{Text Output Display}

For objectives of the system to process regional data statistics, computation, and inference and final assessment, text output will facilitate precise description and understanding. As the system can be used for all counties in China, common text output will be necessary.

\subsubsection{Text Presentation of Statistics and Advantage Computation Results}

In general, the statistics and comparative advantage result can be described by simple text of excellent, good, etc. The following subsection will in particular illustrate the output assessment recommendations.

\subsubsection{Assessment Recommendation Creation and Text Presentation}

According to computation result of some indices, for example, both wheat planting area and rural population at a region are advantageous. It can infer that the region's per capita wheat land area is also advantageous. In this case, its total wheat production is generally advantageous. However, through comparative advantage and ranking, it may not be the case since its total wheat production is less advantageous. 
Under the similar conditions of soil, climate, and other natural resource compared with adjacent regions, there must be some problems probably in wheat species, plantation management, or others.

Based upon the above assumption, a knowledge base is developed in the system to store and compare the computation results, as well as judge rules, and relevant knowledge. The knowledge base also incorporated reasoning mechanisms to make the system have artificial intelligent capability.

For this further system function, based on the advantageous agricultural industry analysis and comparative advantage computation, the knowledge in the base is used to judge and reason the computation result in order to create assessment and action recommendations expressed in text. This can be referred to be knowledge reasoning. Instead of index values, text is used to represent the assessment with expert knowledge and reasoning result.

This is an innovative design and new experiment. So far, the knowledge base is still rich and for further development. However, the knowledge base can be easily maintained and upgraded under the assistance by regional planning, agro-economy, and agricultural experts. According to a partial investigation, knowledge based system or artificial intelligence technology is less used and seldom reported in regional analysis and regional planning applications. Further studies in this aspect are worth strengthening.

\section{Acknowledgements}

This research was supported by National Scientific and Technical Supporting Programs Funded by Ministry of Science and Technology of China (2006BAD10A06, 2006BAD10A12), Special Fund of Basic Scientific Research and Operation Foundation for Commonweal Scientific Research Institutes (2008J-1-06).

\section{Reference}

Fengying, N.: Grain security and its studies. China Agricultural Science and Technology Press, Beijing (2006) (in Chinese) 